\title{
Glycemic variability in relation to oral disposition index in the subjects with different stages of glucose tolerance
}

Tong Chen ${ }^{1 \dagger}$, Feng $\mathrm{Xu}^{2+}$, Jian-bin Su${ }^{2 *}$, Xue-qin Wang ${ }^{2}$, Jin-feng Chen ${ }^{2}$, Gang $\mathrm{Wu}^{2}$, Yan $\mathrm{Jin}^{2}$ and Xiao-hua Wang ${ }^{2}$

\begin{abstract}
Background: Glucose variability could be an independent risk factor for diabetes complications in addition to average glucose. The deficiency in islet $\beta$ cell secretion and insulin sensitivity, the two important pathophysiological mechanisms of diabetes, are responsible for glycemic disorders. The oral disposition index evaluated by product of insulin secretion and sensitivity is a useful marker of islet $\beta$ cell function. The aim of the study is to investigate glycemic variability in relation to oral disposition index in the subjects across a range of glucose tolerance from the normal to overt type 2 diabetes.
\end{abstract}

Methods: $75-\mathrm{g}$ oral glucose tolerance test (OGTT) was performed in total 220 subjects: 47 with normal glucose regulation (NGR), 52 with impaired glucose metabolism (IGM, 8 with isolated impaired fasting glucose [IFG], 18 with isolated impaired glucose tolerance [IGT] and 26 with combined IFG and IGT), 61 screen-diagnosed diabetes by isolated 2-h glucose (DM2h) and 60 newly diagnosed diabetes by both fasting and 2-h glucose (DM). Insulin sensitivity index (Matsuda index, ISI), insulin secretion index $(\Delta \mid 30 / \Delta G 30)$, and integrated $\beta$ cell function measured by the oral disposition index $(\Delta \mathrm{I} 30 / \Delta \mathrm{G} 30$ multiplied by the ISI) were derived from OGTT. All subjects were monitored using the continuous glucose monitoring system for consecutive 72 hours. The multiple parameters of glycemic variability included the standard deviation of blood glucose (SD), mean of blood glucose (MBG), high blood glucose index (HBGl), continuous overlapping net glycemic action calculated every $1 \mathrm{~h}$ (CONGA1), mean of daily differences (MODD) and mean amplitude of glycemic excursions (MAGE).

Results: From the NGR to IGM to DM2h to DM group, the respective values of SD (mean \pm SD) $(0.9 \pm 0.3,1.5 \pm 0.5$, $1.9 \pm 0.6$ and $2.2 \pm 0.6 \mathrm{mmol} / \mathrm{)}, \mathrm{MBG}(5.9 \pm 0.5,6.7 \pm 0.7,7.7 \pm 1.0$ and $8.7 \pm 1.5 \mathrm{mmol} / \mathrm{L}), \mathrm{HGBI}$ [median(Q1-Q3)][0.8 $(0.2-1.2), 2.0(1.2-3.7), 3.8(2.4-5.6)$ and 6.4(3.2-9.5)], CONGA1 $(1.0 \pm 0.2,1.3 \pm 0.2,1.5 \pm 0.3$ and $1.8 \pm 0.4 \mathrm{mmol} / \mathrm{L})$, $\operatorname{MODD}(0.9 \pm 0.3,1.4 \pm 0.4,1.8 \pm 0.7$ and $2.1 \pm 0.7 \mathrm{mmol} / \mathrm{L})$ and MAGE $(2.1 \pm 0.6,3.3 \pm 1.0,4.3 \pm 1.4$ and $4.8 \pm$ $1.6 \mathrm{mmol} / \mathrm{L}$ ) were all increased progressively (all $p<0.05$ ), while their oral disposition indices [745(546-947), 362 (271-475), 203(134-274) and 91(70-139)] were decreased progressively $(p<0.05)$. In addition, SD, MBG, HGBI, CONGA1, MODD and MAGE were all negatively associated with the oral disposition index in each group (all $p<0.05)$ and in the entire data set $(r=-0.66,-0.66,-0.72,-0.59,-0.61$ and -0.65 , respectively, $p<0.05)$.

Conclusions: Increased glycemic variability parameters are consistently associated with decreased oral disposition index in subjects across the range of glucose tolerance from the NGR to IGM to DM2h to DM group.

Keywords: Glycemic variability, Continuous glucose monitoring, Oral disposition index, Type 2 diabetes

\footnotetext{
*Correspondence: sujbzjx@163.com

${ }^{\dagger}$ Equal contributors

${ }^{2}$ Department of Endocrinology, The Second Affiliated Hospital of Nantong University, No. 6 North Hai-er-xiang Road, Chongchuan District, Nantong 226001, China

Full list of author information is available at the end of the article
} 


\section{Background}

The deficiency in islet $\beta$ cell secretion and insulin sensitivity, the two important pathophysiological mechanisms of diabetes, are responsible for disorders of glycemic metabolism [1-3]. Both $\beta$ cell secretion dysfunction and insulin resistance can be demonstrated long before overt diabetes and may differ in the different stages of glucose tolerance from the normal glucose regulation (NGR) via impaired glucose metabolism (IGM) to early screendiagnosed diabetes by 2 -h glucose (DM2h) to overt diabetes by fasting and 2-h glucose (DM).

Insulin secretion and insulin resistance can be quantified with the hyperglycemic and euglycemic insulin clamp techniques, respectively [4]. However, these techniques are labor intensive and are difficult to apply in clinical practices or in large epidemiological studies. Surrogate measures of insulin secretion and insulin sensitivity have been developed from oral glucose tolerance test (OGTT) [5]. The product of insulin secretion and sensitivity derived from OGTT $(\Delta \mathrm{I} 30 / \Delta \mathrm{G} 30 \times \mathrm{ISI})$, also termed oral disposition index, is a useful marker of integrated islet $\beta$ cell function [6]. The oral disposition index as a composite measure may be a better index than either $\Delta \mathrm{I} 30 / \Delta \mathrm{G} 30$ or ISI alone to reflect the notion of declining $\beta$ cell function and account for glycemic deteriorations from the normal to overt diabetes.

Glucose variability could be an independent risk factor for diabetes complications in addition to average glucose [7-9]. The continuous glucose monitoring (CGM) system can detect glycemic variability in more details than the conventional self-monitoring methods of blood glucose $[10,11]$. Glycemic variability parameters, which could be calculated from CGM data [12], may differ in the progression from the normal to overt diabetes.

In this study, we investigated the multiple glycemic variability parameters in relation to oral disposition index in the subjects across a range of glucose tolerance from the NGR to IGM to DM2h to overt DM group.

\section{Methods}

\section{Study subjects}

Total 220 subjects were recruited in this study from January 2012 to January 2013: 47 with normal glucose regulation (NGR), 52 with impaired glucose metabolism (IGM, 8 with isolated impaired fasting glucose [IFG], 18 with isolated impaired glucose tolerance [IGT] and 26 with combined IFG and IGT), 61 screen-diagnosed diabetes by isolated 2-h glucose (DM2h) and 60 newly diagnosed diabetes by both fasting and 2-h glucose (DM). The diagnosis of IFG, IGT and type 2 diabetes were based on the ADA diagnostic criteria 2011 [13]. The oral glucose tolerance test (OGTT) screen program was mainly performed in subjects with diabetes risk factors, such as first-degree relative with diabetes, body mass index $>24 \mathrm{~kg} / \mathrm{m}^{2}$, dyslipidemia, hypertension, et al. Patients with IGM or DM2h were screened and diagnosed by twice 75 -g OGTT. Patients with symptomic hyperglycemia and overt diabetes were diagnosed by once $75-\mathrm{g}$ OGTT. Patients have no acute complications, such as diabetic ketoacidosis, or other disorders affecting glucose metabolism, and did not received anti-diabetic drug treatment. The study was approved by the institutional review board of the Second Affiliated Hospital of Nantong University, with written informed consent being obtained from all participants.

\section{Baseline measurements}

Baseline measurements, including height, weight, and blood pressure, were obtained from all subjects in light clothing and without shoes. Body mass index (BMI) was calculated by dividing weight $(\mathrm{kg})$ by height squared $\left(\mathrm{m}^{2}\right)$. Systolic blood pressure (SBP) and diastolic blood pressure (DBP) were taken three times using a sphygmomanometer and then averaged for further analysis.

\section{$\beta$ cell function determination}

All subjects were examined by the 75 -g oral glucose test. Blood samples were taken at $0,30,60,90$, and $120 \mathrm{~min}$ for the measurement of plasma glucose and insulin concentrations (glucose unit: $\mathrm{mmol} / \mathrm{L}$, insulin unit: miu/L). Insulin sensitivity was estimated using the insulin sensitivity index (ISI) of Matsuda and DeFronzo: ISI $=10,000$ / square root of $(\mathrm{Ins} 0 \times \mathrm{Glu} 0) \times($ mean glucose $\times$ mean insulin during OGTT) [14]. Insulin secretion was estimated by the insulinogenic index calculated from the ratio of increments of serum insulin to glucose measured at $30 \mathrm{~min}$ by the follows: $\Delta \mathrm{I} 30 / \Delta \mathrm{G} 30=(\operatorname{Ins} 30-\mathrm{Ins} 0) /(\mathrm{Glu} 30-\mathrm{Glu} 0)$ [15]. Integrated $\beta$ cell function was measured by the oral disposition index as the product of Insulin secretion and insulin sensitivity $((\Delta \mathrm{I} 30 / \Delta \mathrm{G} 30) \times \mathrm{ISI})[6]$.

\section{Continuous glucose monitoring (CGM) in subjects}

All subjects were monitored by CGM system (Medtronic MiniMed, Northridge, CA 91325, USA) for 72 hours after OGTT. The CGM system sensor was inserted in all patients on day 0 and removed on day 3. Data were downloaded and glucose profiles were evaluated based on the data collected on day 1 and 2 . The patients were instructed to input at least four calibration readings per day and the times of key events. During the study, all subjects had standard meals provided by dietary division. The total calorie intake was $30 \mathrm{kcal} / \mathrm{kg}$ per day, with $50 \%$ carbohydrates, $15 \%$ proteins, and $35 \%$ fats. The calorie distribution between breakfast, lunch, and dinner was $20 \%, 40 \%$, and $40 \%$, respectively. Three daily meals were required to consume at time of $6: 30$ to $7: 30,11: 30$ to $12: 30$, and 18:00 to $19: 00$, respectively. 
Table 1 Clinical characteristics in subjects with different stages of glucose tolerance

\begin{tabular}{|c|c|c|c|c|c|}
\hline & NGR & IGM & DM2h & DM & $P$ \\
\hline$N$ & 47 & 52 & 61 & 60 & - \\
\hline Age(year) & $45 \pm 10$ & $46 \pm 13$ & $48 \pm 13$ & $44 \pm 12$ & 0.093 \\
\hline Female(\%) & $22(46.8)$ & $21(40.4)$ & $26(51.0)$ & $28(46.7)$ & 0.886 \\
\hline $\mathrm{BMI}\left(\mathrm{kg} / \mathrm{m}^{2}\right)$ & $24.8 \pm 3.4$ & $25.2 \pm 2.8$ & $24.7 \pm 2.5$ & $25.0 \pm 4.4$ & 0.182 \\
\hline $\mathrm{SBP}(\mathrm{mmHg})$ & $119 \pm 12$ & $126 \pm 13^{*}$ & $125 \pm 15^{*}$ & $132 \pm 18^{*}$ & 0.001 \\
\hline $\mathrm{DBP}(\mathrm{mmHg})$ & $75 \pm 10$ & $77 \pm 9$ & $79 \pm 10^{*}$ & $80 \pm 8^{*}$ & 0.015 \\
\hline $\mathrm{TG}(\mathrm{mmol} / \mathrm{L})$ & $1.4(0.8-2.2)$ & $1.5(0.9-2.5)$ & $1.9(1.1-3.3)^{*} \neq$ & $1.8(1.4-4.0)^{*} \ddagger$ & 0.002 \\
\hline $\mathrm{TC}(\mathrm{mmol} / \mathrm{L})$ & $4.5 \pm 1.2$ & $4.7 \pm 1.0$ & $5.1 \pm 1.3^{*}$ & $5.3 \pm 2.0^{*} \neq$ & 0.022 \\
\hline $\mathrm{HDL}-\mathrm{C}(\mathrm{mmol} / \mathrm{L})$ & $1.4 \pm 0.4$ & $1.2 \pm 0.3^{*}$ & $1.1 \pm 0.3^{*}$ & $1.1 \pm 0.3^{*}$ & 0.013 \\
\hline $\mathrm{LDL}-\mathrm{C}(\mathrm{mmol} / \mathrm{L})$ & $2.3 \pm 0.8$ & $2.5 \pm 0.8$ & $2.8 \pm 1.0^{*} \neq$ & $2.6 \pm 0.7^{*}$ & 0.020 \\
\hline $\mathrm{HbA} 1 \mathrm{c}(\%)$ & $5.3 \pm 0.5$ & $6.3 \pm 1.1^{*}$ & $7.6 \pm 1.6^{*} \neq$ & $8.9 \pm 1.8^{*} \neq \S$ & 0.000 \\
\hline$|S|$ & $168(122-222)$ & $131(97-203)^{*}$ & $108(74-162)^{*} \neq$ & $92(67-142)^{*} \neq$ & 0.002 \\
\hline$\Delta \mathrm{l} 30 / \Delta \mathrm{G} 30$ & $4.5(3.7-5.8)$ & $2.6(1.4-4.4)^{*}$ & $1.6(1.1-3.1)^{*} \neq$ & $1.0(0.5-1.9)^{*} \neq \S$ & 0.000 \\
\hline oral disposition index & 745(546-947) & $362(271-475)^{*}$ & $203(134-274)^{*} \ddagger$ & $91(70-139)^{*} \neq \S$ & 0.000 \\
\hline $\mathrm{SD}(\mathrm{mmol} / \mathrm{L})$ & $0.9 \pm 0.3$ & $1.5 \pm 0.5^{*}$ & $1.9 \pm 0.6^{*} \neq$ & $2.2 \pm 0.6^{*} \neq \S$ & 0.000 \\
\hline $\mathrm{MBG}(\mathrm{mmol} / \mathrm{L})$ & $5.9 \pm 0.5$ & $6.7 \pm 0.7^{*}$ & $7.7 \pm 1.0^{*} \neq$ & $8.7 \pm 1.5^{*} \neq \S$ & 0.000 \\
\hline $\mathrm{HBGl}$ & $0.8(0.2-1.2)$ & $2.0(1.2-3.7)^{*}$ & $3.8(2.4-5.6)^{*} \neq$ & $6.4(3.2-9.5)^{*} \neq \S$ & 0.000 \\
\hline CONGA1(mmol/L) & $1.0 \pm 0.2$ & $1.3 \pm 0.2^{*}$ & $1.5 \pm 0.3^{*} \neq$ & $1.8 \pm 0.4^{*} \neq \S$ & 0.000 \\
\hline $\mathrm{MODD}(\mathrm{mmol} / \mathrm{L})$ & $0.9 \pm 0.3$ & $1.4 \pm 0.4^{*}$ & $1.8 \pm 0.7^{*} \neq$ & $2.1 \pm 0.7^{*} \neq \S$ & 0.000 \\
\hline MAGE(mmol/L) & $2.1 \pm 0.6$ & $3.3 \pm 1.0^{*}$ & $4.3 \pm 1.4^{*} \neq$ & $4.8 \pm 1.6^{*} \neq \S$ & 0.000 \\
\hline
\end{tabular}

Normally distributed values in the table are given as the mean \pm SD, and the non-normally distributed values are given as the median $(25 \%$ and $75 \%$ interquartiles).

NGR normal glucose regulation, IGM impaired glucose metabolism, DM2h screen-diagnosed diabetes by isolated 2-h glucose, DM newly diagnosed diabetes by both fasting and 2-h glucose.

$B M I$ body mass index, SBP/DBP systolic/diastolic blood pressure, $T C$ total cholesterol, $T G$ triglyceride, $H D L-C$ high density lipoprotein cholesterol, $L D L-C$ low density lipoprotein cholesterol, $H b A 1 c$ glycosylated hemoglobin $A 1 C, I S I$ insulin sensitivity index, $\triangle I 30 / \triangle G 30$ serum insulin to glucose measured at 30 min, $S D$ standard deviation of blood glucose, $M B G$ mean of blood glucose, $H B G l$ high blood glucose index, CONGA1 continuous overlapping net glycemic action calculated every $1 \mathrm{~h}, M O D D$ mean of daily differences, MAGE mean amplitude of glycemic excursions.

Sex distribution was compared by Chi-squared test; comparison to NGR: ${ }^{*} p<0.05$; comparison to IGM: $\neq p<0.05$; comparison to DM2h: $\S p<0.05$.

The parameters of glycemic variability included the standard deviation of blood glucose (SD), mean of daily continuous 24 hours blood glucose (MBG), high blood glucose index (HBGI), continuous overlapping net glycemic action calculated every $1 \mathrm{~h}$ (CONGA1), mean of daily differences (MODD) and mean amplitude of glycemic excursions (MAGE) [12]. Continuous overlapping net glycemic action (CONGA) was calculated by determining the difference between values at different set intervals [16], and CONGA1 was calculated every $1 \mathrm{~h}$ during the monitoring period. High blood glucose index (HBGI) was used to assess the hyperglycemic risk [17]. The mean of daily differences (MODD) was calculated from the absolute difference between paired continuous glucose monitoring values during two successive 24 hour periods and was used to assess inter-day glycemic variability [18]. The mean amplitude of glycemic excursions (MAGE), which was designed to quantify major swings of glycemia and to exclude minor ones, was used for assessing intra-day glycemic variability in this study $[19,20]$. It should be noted that MBG is a

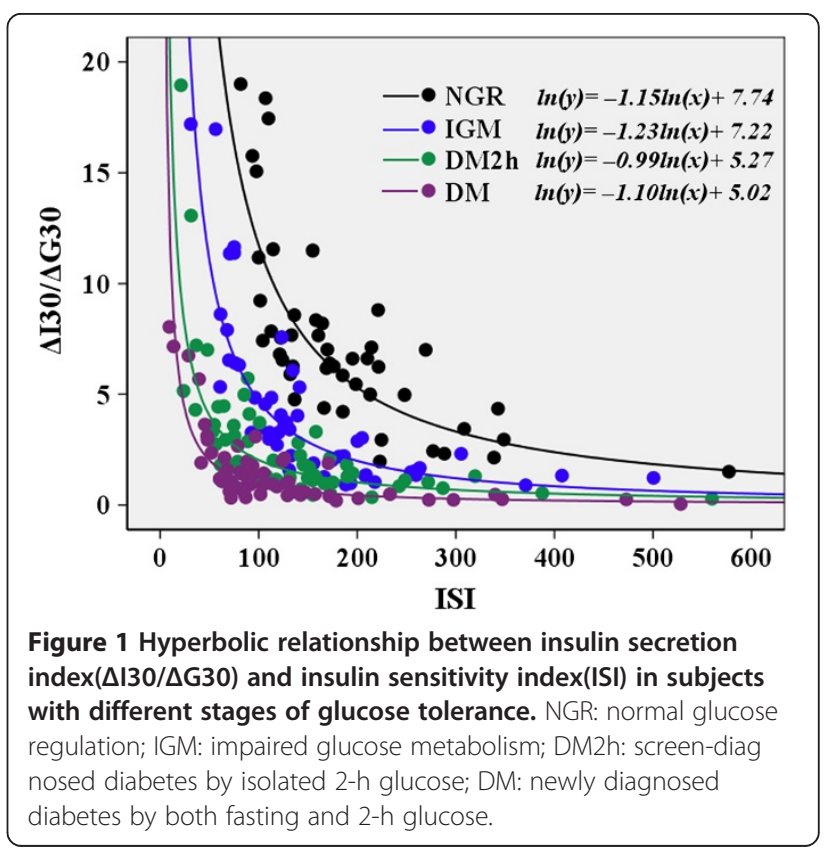


measure of quality of glycemic control and not specifically variability.

\section{Laboratory examination}

Capillary glucose concentrations were measured with Lifescan Surestep blood glucose meter. Glycosylated hemoglobin A1c (HbA1c) was measured by the high performance liquid chromatography (HPLC) with D-10 hemoglobin Testing Program (Bio-Rad). Serum glucose concentrations were measured by the glucose oxidase method. Serum insulin concentrations were measured by magnetic beads-based enzymatic spectrofluorometric immunoassay with automatic enzyme immunoassay apparatus (AIA360, TOSOH). Total cholesterol (TC), triglyceride (TG), high density lipoprotein cholesterol (HDL-C), and low density lipoprotein cholesterol (LDLC) were measured with Hitachi Model 7600 Series Automatic Analyzer.

\section{Statistical analyses}

Data analyses were performed using the SPSS16.0 statistical software (SPSS Inc., USA). Continuous variables were expressed as means \pm standard deviation (SD) or median (interquartile range) in the case of skewed distributions, and sex ratio were expressed as $n$ (\%). Natural logarithmic transformations were applied for all nonnormally distributed variables.

The validity of the oral disposition index was assessed by demonstrating whether a hyperbolic relationship existed between OGTT-based measures of insulin secretion and insulin sensitivity. Regression analysis was applied to combinations of insulin secretion and insulin sensitivity to determine the regression coefficient $\beta$ for the following model: $\ln ($ insulin secretion $)=$ constant $+\beta \times \ln ($ insulin sensitivity). The hyperbolic relationship can be established if estimated $\beta$ is approximately equal to -1 and with $95 \%$ confidence interval (CI) excluding $0[21,22]$

Table 2 Correlation coefficients among the glycemic variability in subjects with different stages of glucose tolerance

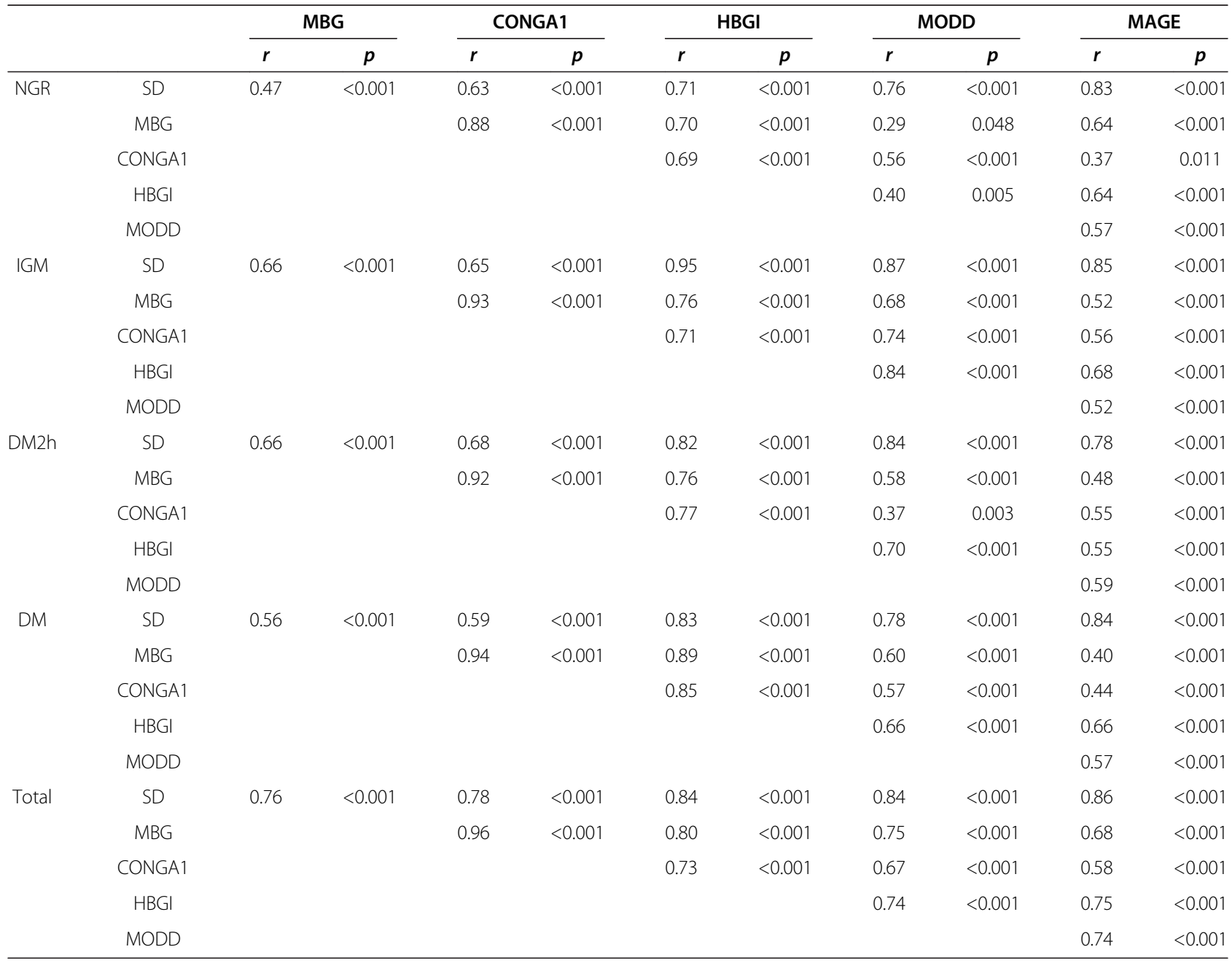

NGR normal glucose regulation, IGM impaired glucose metabolism, DM2h screen-diagnosed diabetes by isolated 2-h glucose, DM newly diagnosed diabetes by both fasting and 2-h glucose, SD standard deviation of blood glucose, MBG mean of blood glucose, HBGl high blood glucose index, CONGA1 continuous overlapping net glycemic action calculated every $1 \mathrm{~h}, M O D D$ mean of daily differences, MAGE mean amplitude of glycemic excursions. 
Table 3 The ratios of MAGE, MODD and CONGA1 to SD in subjects with different stages of glucose tolerance

\begin{tabular}{lcccccc}
\hline & NGR & IGM & DM2h & DM & $P$ & Total \\
\hline MAGE/SD & $2.26 \pm 0.060$ & $2.23 \pm 0.060$ & $2.23 \pm 0.064$ & $2.25 \pm 0.060$ & 0.988 & $2.24 \pm 0.031$ \\
MODD/SD & $0.99 \pm 0.030$ & $0.93 \pm 0.026$ & $0.92 \pm 0.030$ & $0.92 \pm 0.026$ & 0.320 & $0.94 \pm 0.014$ \\
CONGA1/SD & $0.93 \pm 0.02$ & $0.88 \pm 0.02$ & $0.89 \pm 0.02$ & $0.87 \pm 0.03$ & 0.350 & $0.89 \pm 0.01$ \\
\hline
\end{tabular}

The ratios in the table are given as the mean \pm SEM.

NGR normal glucose regulation, IGM impaired glucose metabolism, DM2h screen-diagnosed diabetes by isolated 2-h glucose, $D M$ newly diagnosed diabetes by both fasting and 2-h glucose, SD standard deviation of blood glucose, MBG mean of blood glucose, HBGI high blood glucose index, CONGA1 continuous overlapping net glycemic action calculated every $1 \mathrm{~h}, M O D D$ mean of daily differences, MAGE mean amplitude of glycemic excursions.

The one-way analysis of variance (ANOVA) test were applied to compare differences of continuous variables among groups, and the least significant difference (LSD) test for multiple comparisons was further performed. Chi-squared test was applied to compare sex distribution among groups. Relationships between glycemic variability and oral disposition index were assessed using the Pearson's correlation test. $p<0.05$ was considered to be statistically significant.

\section{Results}

Baseline characteristics of the subjects

As shown in Table 1, age, sex distribution and BMI were comparable among the four groups. The blood pressure (SBP and DBP) in the IGM, DM2h and DM groups were generally higher than those in the NGR group. The lipid metabolic disorders were also observed in the IGM, DM2h and DM groups. The glycosylated hemoglobin A1c (HbA1c) was increased progressively from the NGR to IGM to DM2h to DM group (Table 1).

$\beta$ cell function index derived from OGTT in the subjects Insulin secretion index $(\Delta \mathrm{I} 30 / \Delta \mathrm{G} 30)$ was decreased progressively from the NGR to IGM to DM2h to DM group $(p<0.05)$. Matsuda index (ISI) in the NGR and IGM groups were higher than in DM2h and DM groups $(p<0.05)$, and the DM2h and DM groups had no differences in ISI $(p>0.05)$ (Table 1$)$.
The oral disposition index was decreased progressively from the NGR to IGM to DM2h to DM group $(p<0.01)$ (Table 1). Figure 1 displays the hyperbolic relationship between insulin secretion and insulin sensitivity for each glucose tolerance group using the product of the $\Delta \mathrm{I} 30 /$ $\Delta$ G30 and ISI. The hyperbolic curves demonstrated a shift to the left and downward from the NGR to IGM to DM2h to DM. The corrected slopes included -1 for the relationship between $\ln (\Delta \mathrm{I} 30 / \Delta \mathrm{G} 30)$ and $\ln (\mathrm{ISI})$ in the NGR $(-1.15$ [95\% CI -1.39 to -0.91$])$, IGM $(-1.23$ $[-1.44,-1.03])$, DM2h $(-0.99[-1.19,-0.80])$ and DM $(-1.10[-1.31,-0.90])$. So the validity of the oral disposition index was documented.

\section{Glycemic variability in the subjects}

The multiple glycemic variability parameters from CGM data were shown in Table 1. After comparison within the four groups, SD, MBG, HGBI, CONGA1, MODD and MAGE were all increased progressively from the NGR to IGM to DM2h to DM group $(p<0.05)$.

\section{Inter-relationships among glycemic variability in the subjects}

The Spearman's correlation coefficients among the parameters of glycemic variability were analyzed to measure whether they have agreements in characterizing glycemic variability (Table 2). There are high degrees of agreement among SD, MBG, HGBI, CONGA1, MODD and MAGE, with almost correlations being significant at

Table 4 Relationships between glycemic variability and oral disposition index in subjects with different stages of glucose tolerance

\begin{tabular}{|c|c|c|c|c|c|c|c|c|c|c|}
\hline & \multicolumn{2}{|c|}{ NGR } & \multicolumn{2}{|c|}{ IGM } & \multicolumn{2}{|c|}{$\mathrm{DM} 2 \mathrm{~h}$} & \multicolumn{2}{|c|}{ DM } & \multicolumn{2}{|c|}{ Total } \\
\hline & $r$ & $p$ & $r$ & $p$ & $r$ & $p$ & $r$ & $p$ & $r$ & $p$ \\
\hline SD & -0.29 & 0.048 & -0.38 & 0.006 & -0.36 & 0.005 & -0.45 & $<0.001$ & -0.66 & $<0.001$ \\
\hline MBG & -0.56 & $<0.001$ & -0.35 & 0.012 & -0.42 & 0.001 & -0.40 & 0.001 & -0.66 & $<0.001$ \\
\hline $\mathrm{HBGl}$ & -0.30 & 0.043 & -0.32 & 0.023 & -0.47 & $<0.001$ & -0.47 & $<0.001$ & -0.72 & $<0.001$ \\
\hline CONGA1 & -0.47 & 0.001 & -0.34 & 0.015 & -0.43 & 0.001 & -0.32 & 0.014 & -0.59 & $<0.001$ \\
\hline MODD & -0.33 & 0.022 & -0.48 & $<0.001$ & -0.30 & 0.018 & -0.41 & 0.001 & -0.61 & $<0.001$ \\
\hline MAGE & -0.55 & $<0.001$ & -0.41 & 0.003 & -0.34 & 0.007 & -0.40 & 0.002 & -0.65 & $<0.001$ \\
\hline
\end{tabular}

$\mathrm{HBGI}$ and oral disposition index were In-transformed.

NGR normal glucose regulation, IGM impaired glucose metabolism, DM2h, screen-diagnosed diabetes by isolated 2-h glucose, DM newly diagnosed diabetes by both fasting and 2-h glucose, SD standard deviation of blood glucose, MBG mean of blood glucose, HBGl high blood glucose index, CONGA1 continuous overlapping net glycemic action calculated every $1 \mathrm{~h}$, MODD mean of daily differences, MAGE mean amplitude of glycemic excursions. 

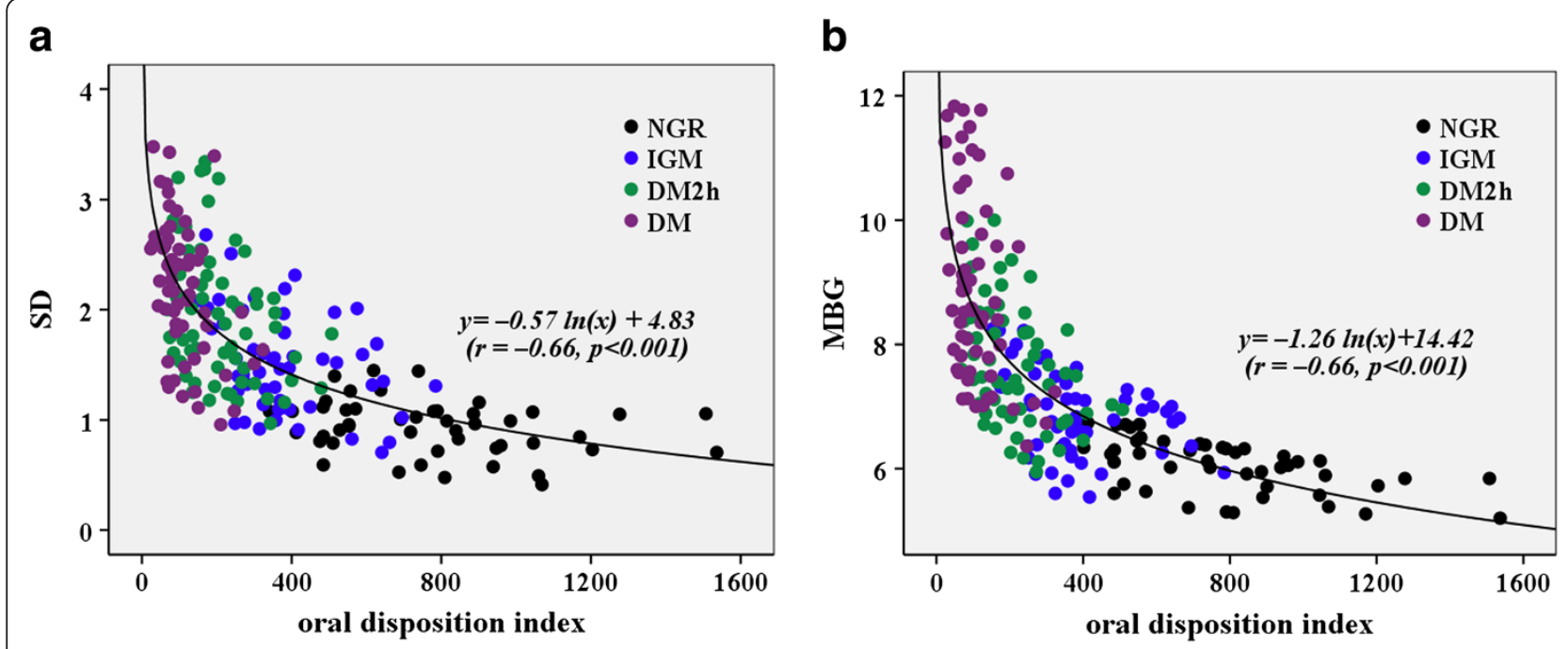

C

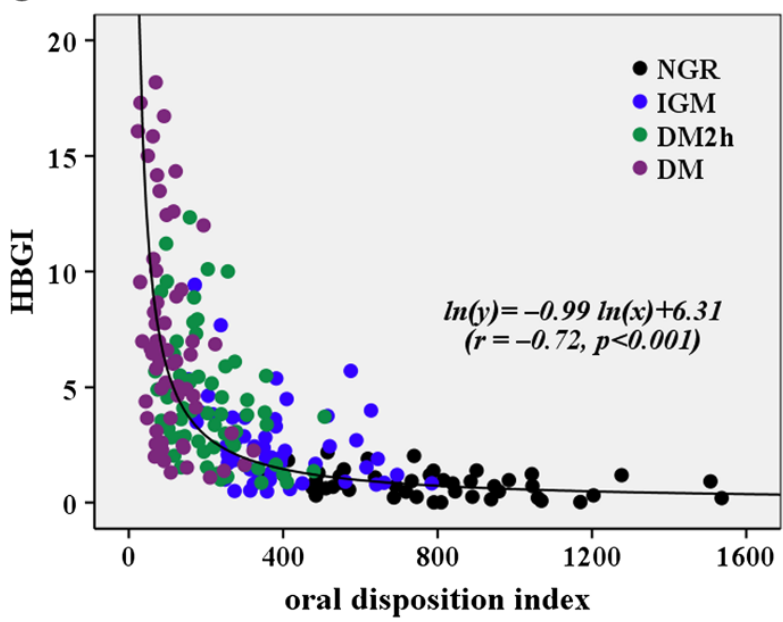

d

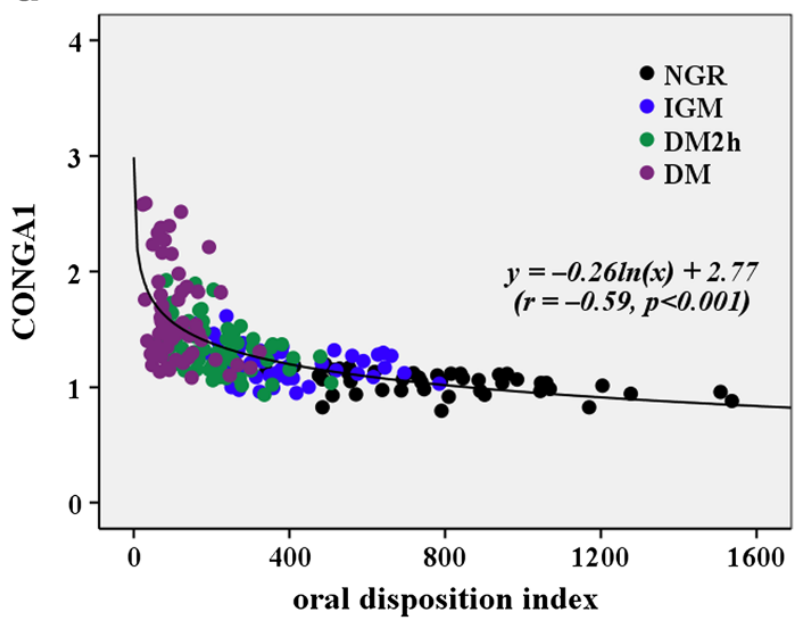

e

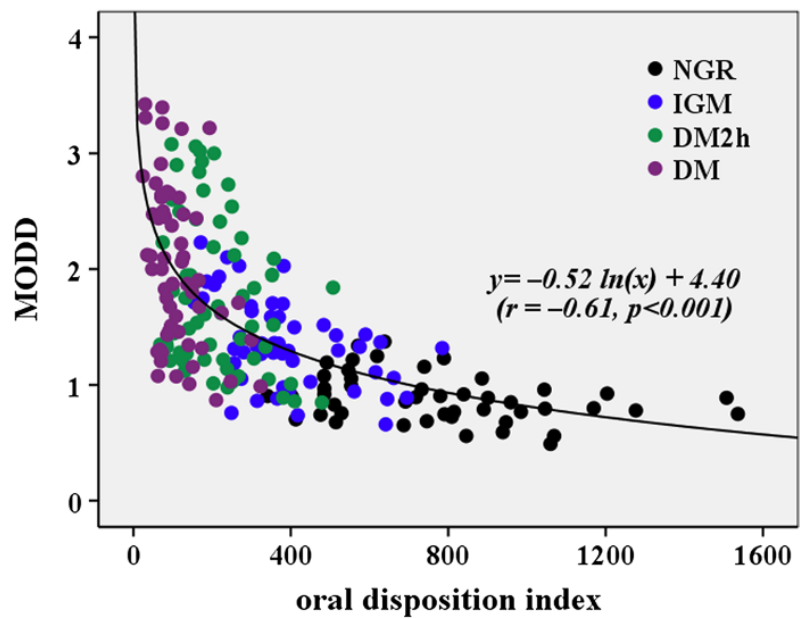

f

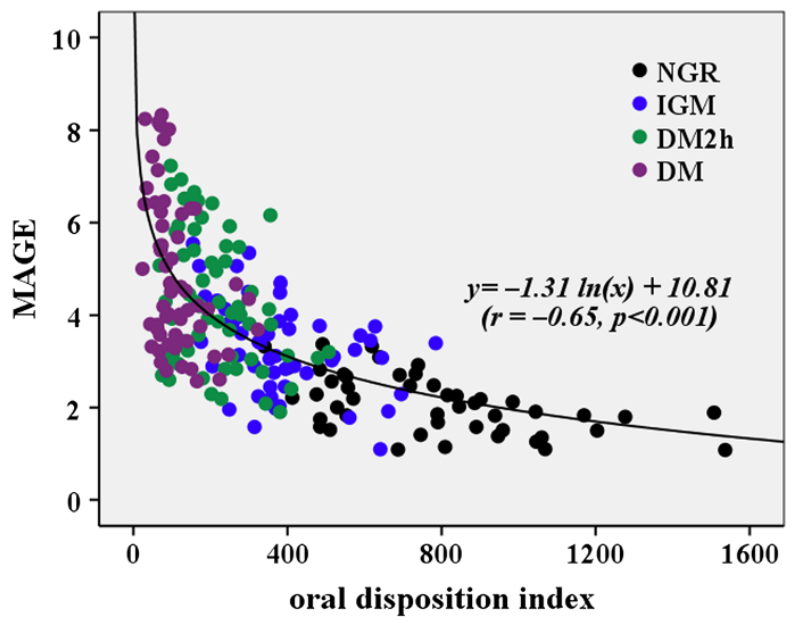

Figure 2 (See legend on next page.) 
(See figure on previous page.)

Figure 2 The overall best-fit lines obtained by nonlinear regression analysis between glycemic variability parameters (a: SD, b: MBG, c: HGBI, d: CONGA1, e: MODD and f: MAGE) and oral disposition index. NGR: normal glucose regulation; IGM: impaired glucose metabolism; DM2h: screen-diagnosed diabetes by isolated 2-h glucose; DM: newly diagnosed diabetes by both fasting and 2-h glucose. SD: standard deviation of blood glucose; MBG: mean of blood glucose; HBGl: high blood glucose index; CONGA1: continuous overlapping net glycemic action calculated every 1 h; MODD: mean of daily differences; MAGE: mean amplitude of glycemic excursions.

the $p<0.01$ level in each glucose tolerance group and in the entire data set.

In the majority of published studies, the standard deviation (SD) around the mean glucose value was considered as a classical and well-validated index to assess the glycemic variability. The ratios of MAGE, MODD and CONGA1 to SD were also analyzed in the study subjects. There were direct linear proportionalities between MAGE, MODD, CONGA1 and SD for the each glucose tolerance group or the entire data set. The ratios of MAGE, MODD and CONGA1 to SD were $2.24 \pm 0.031$ (SEM), $0.94 \pm 0.014$ and $0.89 \pm 0.01$, respectively, in the entire data set. And the ratios of MAGE/SD, MODD/SD and CONGA1/SD were comparable in the NGR, IGM, DM2h and DM groups (ANOVA $p=0.988, p=0.320$ and 0.350 , respectively) (Table 3 ).

\section{Relationships between glycemic variability and oral disposition index}

When the relationships between glycemic variability parameters and oral disposition index were analyzed by Pearson's correlation test, SD, MBG, HGBI, CONGA1, MODD and MAGE were all negatively associated with oral disposition index in each group $(p<0.05)$ and in the entire data set $(p<0.05)$ (Table 4$)$. These relationships remained significant after adjusting for age, sex, BMI, SBP, DBP, TG, TC, HDLC, LDLC and HbA1c.

The overall best-fit lines obtained by nonlinear regression analysis (logarithmic regression) between glycemic variability parameters and oral disposition index are presented in the Figure $2 \mathrm{a}-\mathrm{f}$. The overall regression line between $\mathrm{SD}$ and oral disposition index was $\mathrm{y}=-0.57 \ln (\mathrm{x})+4.83(r=-0.66, p<0.001)$, overall regression line between MBG and oral disposition index was $\mathrm{y}=-1.26 \ln (\mathrm{x})+14.42(r=-0.66, p<0.001)$, overall regression line between HBGI and oral disposition index was $\ln (\mathrm{y})=-0.99 \ln (\mathrm{x})+6.31(r=-0.72$, $p<0.001$ ), overall regression line between CONGA1 and oral disposition index was $\mathrm{y}=-0.26 \ln (\mathrm{x})+2.77$ $(r=-0.59, p<0.001)$, overall regression line between MODD and oral disposition index was $\mathrm{y}=-0.52 \ln (\mathrm{x})+$ $4.40 \quad(r=-0.61, \quad p<0.001)$, overall regression line between MAGE and oral disposition index was $\mathrm{y}=-1.31 \ln (\mathrm{x})+10.81(r=-0.65, \quad p<0.001)$. The correlation coefficients were almost in agreements (Figure 2a-f).

\section{Discussion}

The glycemic disorders in diabetes are not solely limited to fasting and postprandial hyperglycemia, but can be extended to the glycemic variability that includes both upward (postprandial glucose increments) and downward (interprandial glucose decrements) changes [23]. Glucose variability could be an independent risk factor for diabetes complications in addition to average glucose [7-9]. Glucose fluctuations are presented not only in diabetes patients but also in normoglycemic and prediabetes subjects [24], and the characteristics of glucose metabolic disorders may differ in the progression from normal to overt diabetes. Our study recruited the subjects across a range of glucose tolerance from the NGR to IGM to DM2h to overt DM group. The results of our study showed glycemic variability parameters, including SD, MBG, HGBI, CONGA1, MODD and MAGE, increased progressively from the NGR to IGM to DM2h to DM group. The results of our study are consistent with those of Wang et al. [25]. Wang et al. showed the diabetes patients had increased postprandial glucose excursion, higher glucose levels overnight and greater inter-day fluctuations compared with the normoglycemic and impaired glucose regulation individuals. Our findings could have a major impact on our understanding of the overall glycemic variability changes from the NGR to IGM to DM2h to overt DM group and how this could influence different complication consequences.

In addition to metabolic differences, other factors such as meals consumed and drug treatment may be partly responsible for the glycemic variability [26]. We employed a fixed meal regimen in the study and recruited diabetes patients (DM2h and DM groups) who had not received anti-diabetic drug treatment thereby controlling for the dietary and drug effect on glycemic variability. Thus, changes of glycemic variability due to metabolic differences from the normal to overt diabetes were well demonstrated in our study.

The inter-relationships among measures of variability were further assessed to weigh whether they have agreement in characterizing glycemic variability. The results documented that the agreements among the measures were at high degree in each glucose tolerance group and in the entire data set. The results were consistent with those from Hill et al. [11] and Kohnert et al. [27]. In the majority of published studies, the SD around the mean glucose value was considered as a classical and well- 
validated index to assess the glycemic variability [28]. The ratios of MAGE, MODD and CONGA1 to SD were $2.24 \pm 0.031$ (SEM), $0.94 \pm 0.014$ and $0.89 \pm 0.01$, respectively, in the entire data set. The ratios that we have identified fit well with previously reported values of Rodbard et al. [28-30], Fritzsche et al. [31] and Kohnert et al. [27]. The ratio of MAGE/SD was also comparable in NGR, IGM, DM2h and DM groups. And the ratios of MODD/SD and CONGA1/SD were also similar in the four groups. Thus, our findings imply that SD, CONGA1, MODD and MAGE could be used as validated indices to assess the glycemic variability in subjects with different stages of glucose tolerance.

The progression from the NGR via IGM to DM2h to overt diabetes was considered due to the deterioration of insulin secretion and increasing of insulin insensitivity. Insulin secretion index $(\Delta \mathrm{I} 30 / \Delta \mathrm{G} 30)$, a surrogate marker of early insulin response, has been validated against gold-standard measures of insulin secretion obtained from intravenous glucose tolerance testing [32]. The decreased insulin secretion capacity plays a definite role in the development of type 2 diabetes [33,34]. Matsuda index, a marker of whole-body insulin sensitivity, is highly correlated with the rate of whole-body glucose disposal during the euglycemic insulin clamp [14]. Kim et al. [6] found that increased risk for type 2 diabetes was evidenced by a lower oral disposition index $(\Delta \mathrm{I} 30 /$ $\Delta \mathrm{G} 30 \times \mathrm{ISI})$. Our study demonstrated that the oral disposition index declined progressively from the NGR to IGM (IFG/IGT) to early screen-diagnosed diabetes by isolated $2-\mathrm{h}$ post-challenge glucose to overt diabetes by fasting and 2-h glucose. The hyperbolic curves representing the oral disposition index are shifted to the left and downward from the NGR to IGM to DM2h to DM (Figure 1). This shift is also a hallmark of type 2 diabetes pathophysiology and is considered one of the earliest indicators of $\beta$-cell dysfunction [35].

After correlation analysis, SD, MBG, HGBI, CONGA 1, MODD and MAGE were all negatively associated with the oral disposition index in the separate group (NGR, IGM, DM2h or DM) and in the entire data set. These findings imply that diabetic treatment aims to improve $\beta$ cell function may flat glycemic variability.

It should be pointed out that our study has some limitations. The IGM group should be theoretically divided into subgroups with isolated IFG or IGT, but the small sample size of subgroups might make some differences insignificant. We put them into one study group considering that IFG and IGT are intermediate states that exist between normal glucose tolerance and overt diabetes. Another limitation related to oral disposition index is that circulating insulin levels during the OGTT may be affected by other factors apart from $\beta$ cell function, such as incretin hormones and hepatic extraction. The two factors may limit the degree to which insulin levels during the OGTT can reflect $\beta$ cell function. But the validity of the oral disposition index had been assessed by demonstrating that the hyperbolic relationship existed between OGTT-based measures of insulin secretion and insulin sensitivity (Figure 1).

\section{Conclusion}

In summary, our study has demonstrated that increased glycemic variability parameters are consistently associated with decreased oral disposition index in the subjects across the range of glucose tolerance from the NGR to IGM to DM2h to DM group, which not only provides important clues for clinics but also forms a strong basis for further investigations of glycemic disorders.

\section{Abbreviations}

NGR: Normal glucose regulation; IGM: Impaired glucose metabolism; DM2h: Screen-diagnosed diabetes by isolated 2-h glucose; DM: Newly diagnosed diabetes by both fasting and 2-h glucose; BMl: Body mass index; SBP/DBP: Systolic/diastolic blood pressure; TC: Total cholesterol; TG: Triglyceride; HDL-C: High density lipoprotein cholesterol; LDL-C: Low density lipoprotein cholesterol; HbA1c: Glycosylated hemoglobin A1c; ISI: Insulin sensitivity index; $\Delta \mid 30 / \Delta G 30$ : Serum insulin to glucose measured at 30 min; SD: Standard deviation of blood glucose; MBG: Mean of blood glucose; HBGl: High blood glucose index; CONGA1: Continuous overlapping net glycemic action calculated every 1 h; MODD: Mean of daily differences; MAGE: Mean amplitude of glycemic excursions.

\section{Competing interests}

The authors have no competing interests to declare.

\section{Authors' contributions}

TC and FX participated in the design of the study, data collection, analysis of the data, drafting of the manuscript. JS conceived of the study, participated in its design and revised the manuscript. XW participated in analysis of the data and revised the manuscript. JC, GW, YJ and XW participated in data collection. All authors read and approved the final manuscript.

\section{Funding}

The study was funded by the Scientific Research Program of Nantong (No. HS2012028)

\section{Author details}

'Department of Clinical Laboratory, The Second Affiliated Hospital of Nantong University, No. 6 North Hai-er-xiang Road, Chongchuan District, Nantong 226001, China. ${ }^{2}$ Department of Endocrinology, The Second Affiliated Hospital of Nantong University, No. 6 North Hai-er-xiang Road, Chongchuan District, Nantong 226001, China.

Received: 5 May 2013 Accepted: 21 July 2013

Published: 23 July 2013

\section{References}

1. Abdul-Ghani MA, Williams K, DeFronzo R, Stern M: Risk of progression to type 2 diabetes based on relationship between postload plasma glucose and fasting plasma glucose. Diabetes Care 2006, 29:1613-1618.

2. Abdul-Ghani MA, Tripathy D, DeFronzo RA: Contributions of beta-cell dysfunction and insulin resistance to the pathogenesis of impaired glucose tolerance and impaired fasting glucose. Diabetes Care 2006 29:1130-1139.

3. Festa A, Williams K, Hanley AJ, Haffner SM: Beta-cell dysfunction in subjects with impaired glucose tolerance and early type 2 diabetes: comparison of surrogate markers with first-phase insulin secretion from an intravenous glucose tolerance test. Diabetes 2008, 57:1638-1644.

4. Abdul-Ghani MA, Williams K, DeFronzo RA, Stern M: What is the best predictor of future type 2 diabetes? Diabetes Care 2007, 30:1544-1548. 
5. Stumvoll M, Mitrakou A, Pimenta W, Jenssen $T$, Yki-Järvinen $H$, Van Haeften T, Renn W, Gerich J: Use of the oral glucose tolerance test to assess insulin release and insulin sensitivity. Diabetes Care 2000, 23:295-301.

6. Kim JY, Coletta DK, Mandarino LJ, Shaibi GQ: Glucose response curve and type 2 diabetes risk in Latino adolescents. Diabetes Care 2012, 35:1925-1930.

7. Monnier L, Mas E, Ginet C, Michel F, Villon L, Cristol JP, Colette C: Activation of oxidative stress by acute glucose fluctuations compared with sustained chronic hyperglycemia in patients with type 2 diabetes. JAMA 2006, 295:1681-1687.

8. Torimoto K, Okada Y, Mori H, Tanaka Y: Relationship between fluctuations in glucose levels measured by continuous glucose monitoring and vascular endothelial dysfunction in type 2 diabetes mellitus. Cardiovasc Diabetol 2013, 12:1.

9. Sartore G, Chilelli NC, Burlina S, Lapolla A: Association between glucose variability as assessed by continuous glucose monitoring (CGM) and diabetic retinopathy in type 1 and type 2 diabetes. Acta Diabetol 2013, 50:437-442.

10. Klonoff DC, Buckingham B, Christiansen JS, Montori VM, Tamborlane WV, Vigersky RA, Wolpert H, Endocrine Society: Continuous glucose monitoring: an Endocrine Society Clinical Practice Guideline. J Clin Endocrinol Metab 2011, 96:2968-2979.

11. Chinese Diabetes Society: Chinese clinical guideline for continuous glucose monitoring (2012). Chin Med J (Engl) 2012, 125:4167-4174

12. Hill NR, Oliver NS, Choudhary P, Levy JC, Hindmarsh P, Matthews DR: Normal reference range for mean tissue glucose and glycemic variability derived from continuous glucose monitoring for subjects without diabetes in different ethnic groups. Diabetes Technol Ther 2011, 13:921-928.

13. American Diabetes Association: Diagnosis and classification of diabetes mellitus. Diabetes Care 2011, 34:S62-S69.

14. Matsuda M, DeFronzo RA: Insulin sensitivity indices obtained from oral glucose tolerance testing: comparison with the euglycemic insulin clamp. Diabetes Care 1999, 22:1462-1470.

15. Oka R, Yagi K, Sakurai M, Nakamura K, Moriuchi T, Miyamoto S, Nohara A, Kawashiri MA, Takeda Y, Yamagishi M: Insulin secretion and insulin sensitivity on the oral glucose tolerance test (OGTT) in middle-aged Japanese. Endocr J 2012, 59:55-64.

16. McDonnell CM, Donath SM, Vidmar SI, Werther GA, Cameron FJ: A nove approach to continuous glucose analysis utilizing glycemic variation. Diabetes Technol Ther 2005, 7:253-263.

17. Kovatchev BP, Cox DJ, Kumar A, Gonder-Frederick L, Clarke WL: Algorithmic evaluation of metabolic control and risk of severe hypoglycemia in type 1 and type 2 diabetes using self-monitoring blood glucose data. Diabetes Technol Ther 2003, 5:817-828.

18. Molnar GD, Taylor WF, Ho MM: Day-to-day variation of continuously monitored glycaemia: a further measure of diabetic instability. Diabetologia 1972, 8:342-348.

19. Service FJ, Molnar GD, Rosevear JW, Ackerman E, Gatewood LC, Taylor WF: Mean amplitude of glycemic excursions, a measure of diabetic instability. Diabetes 1970, 19:644-655.

20. Zhou J, Li H, Ran X, Yang W, Li Q, Peng Y, Li Y, Gao X, Luan X, Wang W, Jia $W$ : Establishment of normal reference ranges for glycemic variability in Chinese subjects using continuous glucose monitoring. Med Sci Monit 2011, 17:CR9-CR13.

21. Utzschneider KM, Prigeon RL, Faulenbach MV, Tong J, Carr DB, Boyko EJ, Leonetti DL, McNeely MJ, Fujimoto WY, Kahn SE: Oral disposition index predicts the development of future diabetes above and beyond fasting and 2-h glucose levels. Diabetes Care 2009, 32:335-341.

22. Retnakaran R, Shen S, Hanley AJ, Vuksan V, Hamilton JK, Zinman B: Hyperbolic relationship between insulin secretion and sensitivity on oral glucose tolerance test. Obesity (Silver Spring) 2008, 16(8):1901-1907.

23. Monnier L, Colette C, Owens DR: Glycemic variability: the third component of the dysglycemia in diabetes. Is it important? How to measure it? J Diabetes Sci Technol 2008, 2:1094-1100.

24. Borg R, Kuenen JC, Carstensen B, Zheng H, Nathan DM, Heine RJ, Nerup J, BorchJohnsen K, Witte DR, ADAG Study Group: Real-life glycaemic profiles in nondiabetic individuals with low fasting glucose and normal $\mathrm{HbA1c}$ : the $\mathrm{A} 1 \mathrm{C}$ Derived Average Glucose (ADAG) study. Diabetologia 2010, 53:1608-1611.

25. Wang C, Lv L, Yang Y, Chen D, Liu G, Chen L, Song Y, He L, Li X, Tian H, Jia W, Ran X: Glucose fluctuations in subjects with normal glucose tolerance, impaired glucose regulation and newly diagnosed type 2 diabetes mellitus. Clin Endocrinol (Oxf) 2012, 76:810-815.
26. Kildegaard J, Christensen TF, Hejlesen OK: Sources of glycemic variabilitywhat type of technology is needed? J Diabetes Sci Technol 2009, 3:986-991.

27. Kohnert KD, Heinke P, Fritzsche G, Vogt L, Augstein P, Salzsieder E: Evaluation of the mean absolute glucose change as a measure of glycemic variability using continuous glucose monitoring data. Diabetes Technol Ther 2013, 15:448-454.

28. Rodbard D: New and improved methods to characterize glycemic variability using continuous glucose monitoring. Diabetes Technol Ther 2009, 11:551-565.

29. Rodbard D, Bailey T, Jovanovic L, Zisser H, Kaplan R, Garg SK: Improved quality of glycemic control and reduced glycemic variability with use of continuous glucose monitoring. Diabetes Technol Ther 2009, 11:717-723.

30. Rodbard D: Interpretation of continuous glucose monitoring data: glycemic variability and quality of glycemic control. Diabetes Technol Ther 2009, 11:S55-S67.

31. Fritzsche $G$, Kohnert KD, Heinke P, Vogt $L$, Salzsieder E: The use of a computer program to calculate the mean amplitude of glycemic excursions. Diabetes Technol Ther 2011, 13:319-325.

32. Pratley RE, Weyer $C$ : The role of impaired early insulin secretion in the pathogenesis of Type II diabetes mellitus. Diabetologia 2001, 44:929-945.

33. Fukushima M, Suzuki $H$, Seino $Y$ : Insulin secretion capacity in the development from normal glucose tolerance to type 2 diabetes. Diabetes Res Clin Pract 2004, 66:S37-S43.

34. Del Prato S: Loss of early insulin secretion leads to postprandial hyperglycaemia. Diabetologia 2003, 46:M2-M8.

35. Bergman RN: Orchestration of glucose homeostasis: from a small acorn to the California oak. Diabetes 2007, 56:1489-1501.

doi:10.1186/1758-5996-5-38

Cite this article as: Chen et al:: Glycemic variability in relation to oral disposition index in the subjects with different stages of glucose tolerance. Diabetology \& Metabolic Syndrome 2013 5:38.

\section{Submit your next manuscript to BioMed Central and take full advantage of:}

- Convenient online submission

- Thorough peer review

- No space constraints or color figure charges

- Immediate publication on acceptance

- Inclusion in PubMed, CAS, Scopus and Google Scholar

- Research which is freely available for redistribution

Submit your manuscript at www.biomedcentral.com/submit
C) Biomed Central 